\title{
A round Robin-Highliting on the passivating contact technology
}

\author{
Tobias Fellmeth ${ }^{1, *}$, Frank Feldmann ${ }^{1}$, Bernd Steinhauser ${ }^{1}$, Henning Nagel $^{1}$, Sebastian Mack ${ }^{1}$, Martin Hermle ${ }^{1}$, \\ Frank Torregrosa ${ }^{2}$, Andrea Ingenito ${ }^{3}$, Franz-Josef Haug $^{3}$, Audrey Morisset ${ }^{3}$, Florian Buchholz ${ }^{4}$, Aditya Chaudhary ${ }^{4}$, \\ Thibaut Desrues ${ }^{5}$, Felix Haase ${ }^{6}$, Byungsul Min $^{6}$, Robby Peibst ${ }^{6}$, and Loic Tous ${ }^{7}$ \\ ${ }^{1}$ Fraunhofer Institute for Solar Energy Systems (ISE), Heidenhofstr. 2, 79110 Freiburg, Germany \\ ${ }^{2}$ Ion Beam Services (IBS), Rue Gaston Imbert Prolongée, ZI Rousset-Peynier, 13790 Peynier, France \\ ${ }^{3}$ Ecole Polytechnique Fédérale de Lausanne (EPFL), Bätiment 3316 Station 1, 1015 Lausanne, Switzerland \\ ${ }^{4}$ International Solar Energy Research Center e.V (ISC), Rudolf-Diesel-Str. 15, 78467 Konstanz, Germany \\ ${ }^{5}$ Univ Grenoble Alpes, CEA, LITEN, DTS, INES, 50 avenue du lac Léman, 73375 Le Bourget du Lac, France \\ ${ }^{6}$ Institute for Solar Energy Research Hamelin (ISFH), Am Ohrberg 1, 31860 Emmerthal, Germany \\ ${ }^{7}$ Interuniversitair Micro-Electronica Centrum vzw (IMEC), Kapeldreef 75, 3001 Leuven, Belgium
}

Received: 1 July 2021 / Received in final form: 22 October 2021 / Accepted: 15 November 2021

\begin{abstract}
The aim of this work is to demonstrate the maturity of the TOPCon technology by conducting a round-robin on symmetrically processed lifetime samples in the leading European PV institutes EPFL, ISC, CEA-INES, ISFH, IMEC and Fraunhofer ISE within the H2020 funded project called HighLite. For all layers, dark saturation current-densities ranging between 2 and $10 \mathrm{fA} / \mathrm{cm}^{2}$ can be reported. Simultaneously, no metal induced recombination for the two lower sintering temperatures have been observed pointing towards a true passivated contact. Furthermore, contact resistivities below $10 \mathrm{~m} \Omega \mathrm{cm}^{2}$ have been achieved. It seems that the industrial passivating contact matured to a fully passivated and conducting contact enabling full efficiency potential. The fact that this can be realized using either PECVD or LPCVD from various manufacturer is expected to drive costs down and contribute to the increased adoption of the TOPCon technology.
\end{abstract}

Keywords: Silicon solar cell / passivated contact / TOPCon / POLO / round robin

\section{Introduction}

The poly-Si based surface passivation using a thin tunnel oxide also known as "TOPCon" [1] or "POLO" [2] (for simplicity, in the following only the expression "TOPCon" is used) is subjected to an increasing interest from the industry. In the last years, increasing conversion efficiencies were reported ending so far in record values of $24.6 \%$ reached by Trinasolar [3] and recently of $25.25 \%$ reported by Jinkosolar both in lab scale production.

The aim of this work is to demonstrate the maturity of the TOPCon technology by conducting a round-robin in the leading European PV institutes EPFL, ISC, CEAINES, ISFH, IMEC and Fraunhofer ISE within the H2020 funded project called HighLite. Deposition tools from various companies using plasma enhanced chemical vapour deposition PECVD and low-pressure chemical vapour deposition LPCVD were used. Properties of the layers and information of the tools are given in Table 1.

\footnotetext{
* e-mail: tobias.fellmeth@ise.fhg.de
}

\section{Approach}

N-type Cz-Si wafers were saw damage etched by ISC Konstanz and distributed to the mentioned institutes to use their best-known deposition technology to create n-type TOPCon layers on both sides of the wafers. Despite the differences in the deposition technology, all layers consist of a thin tunnel oxide layer between the c-Si absorber and a phosphorus doped poly-Si layer, which was annealed or $\mathrm{POCl}_{3}$-diffused to transform a- to poly-Si and subsequently capped with a hydrogen-rich $\mathrm{SiN}_{x}$ layer. Then, the samples were sent to Fraunhofer ISE for single side screen-printing of a parallel finger pattern with varying finger pitch consisting of nine individual fields (Fig. 1) [4], contact firing at varying temperatures and characterization. The dark saturation current-density for a single, unmetallized TOPCon layer $J_{0 \mathrm{~T}}$ and the total dark saturation current-density for the whole stack consisting of two TOPCon layers and bulk $J_{0}$ for metallized and unmetallized fields (Fig. 2) were extracted by a combination of the QSSPC [5] $J_{0 \mathrm{e}}$ analysis from Kimmerle et al. [6] and the modulated photoluminescence Mod-PL [7] 


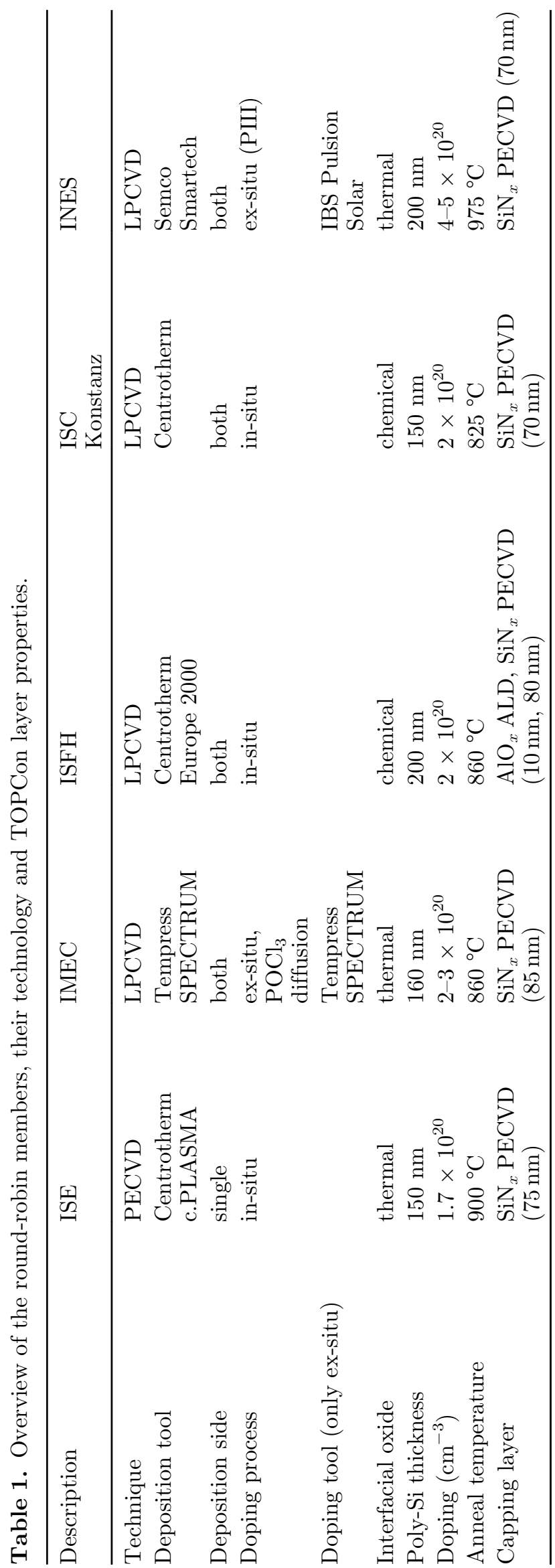

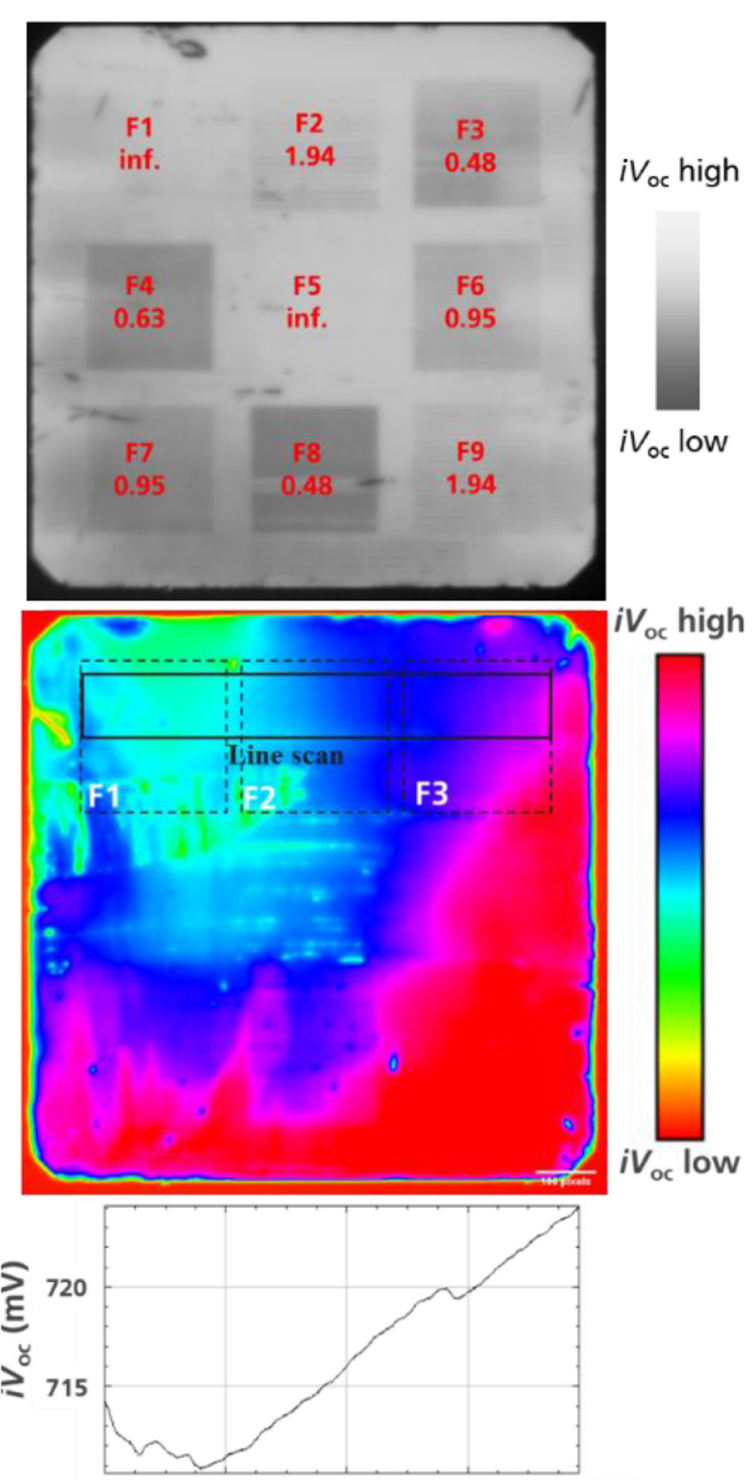

Fig. 1. TOP: A PL image of a over fired wafer, indicating nine individual fields F1-F9 with varying Finger pitches in "mm". On the edges TLM structures are located. Middle: An implied $i V_{\mathrm{oc}}$ Mod-PL image of a selected sample showing no field pattern, but other significant inhomogeneities. The $i V_{\mathrm{oc}}$ as a result from the indicated line scan area is shown in the bottom graph.

method. Finally, transition line method TLM [8] line structures (equidistant lines) were cut off the wafers to obtain the contact resistivity $\rho_{\mathrm{c}}$ (Fig. 3).

All poly-Si layers investigated here exhibiting a thickness ranging from 150 to $200 \mathrm{~nm}$ being in a typical range for screen-printed contacts with sufficiently low parasitic absorption, if embodied as rear electrode [9].

In Table 1, an overview of the round robin members, their technology and layer properties are given. As can be seen, two CVD technologies from three different companies on five different tools are part of the round-robin. 

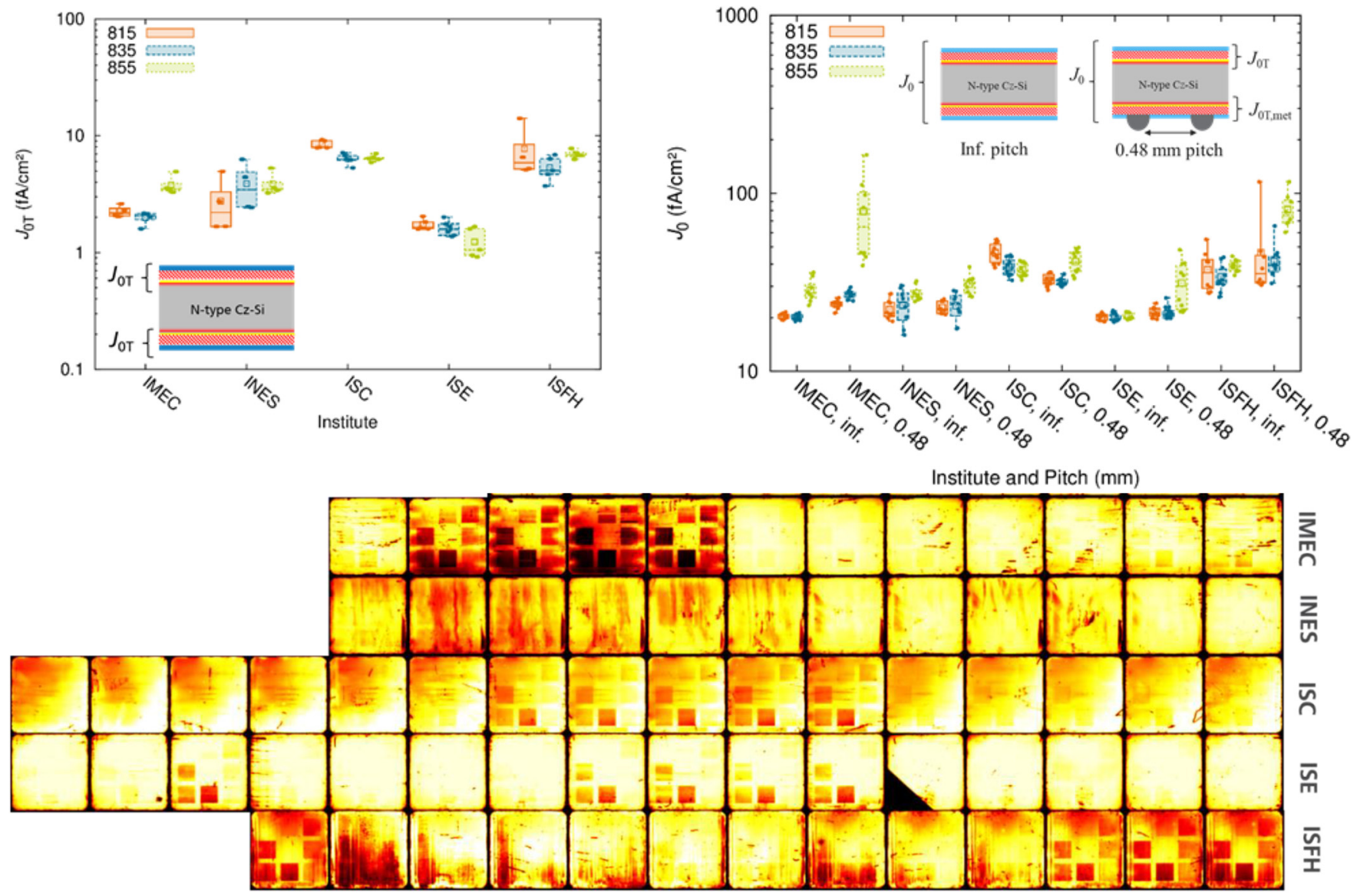

Fig. 2. Upper left graph: Dark saturation density $J_{0 \mathrm{~T}}$ from one single TOPCon layer extracted from field F5. Upper right graph: Total dark recombination current-density $J_{0}$ either consisting of a bulk and two unmetallized TOPCon layers "inf." (average F1 \& F5) or a bulk and two TOPCon layers of which one side is being metallized $(0.48 \mathrm{~mm}$ pitch, average F3 \& F8). Lower part: PL images of all specimens from all institutes showing partly no increased recombination or significant inhomogeneities of which both preventing a local $J_{0 \mathrm{~T}, \mathrm{met}}$ analysis.

In Figure 1, a PL image is shown at the top exhibiting nine individual fields F1-F9 [4]. The fields F1 and F5 serve as reference without metallization, their size being compatible to allow QSSPC measurements. The remaining fields exhibit a parallel allocated finger pattern with nominal finger width of $40 \mu \mathrm{m}$ and as indicated varying finger pitch in "mm".

The middle image of Figure 1 shows a selected $i V_{\text {oc }}$ calibrated PL image to visualize a challenge the authors observed for some samples during the characterization process. At the bottom, a line scan as indicated in the middle image show an increasing $i V_{\mathrm{oc}}$ from field $\mathrm{F} 1$ to $\mathrm{F} 3$. However, one expects a decreasing $i V_{\text {oc }}$ considering the increasing metallized area potentially inducing additional recombination. That seems not to be the case, which points toward a vanishing contact recombination leading to other contrast drivers such as process inhomogeneities.

Additionally, in the cases of over fired samples (see also Fig. 2, PL montage), fields with nominal equal finger count appear different in terms of contrast. That is linked to a slightly inhomogeneous lateral temperature distribution during firing. At set peak temperature of $855^{\circ} \mathrm{C}$, metal induced recombination is on-setting showing an exponential sensitivity on incremental changes in local temperature. This can be seen also in Figure 2 upper right graph where an increasing $J_{0}$ comes along with an increasing spread in data.

\section{Results}

In Figure 2 upper left, the $J_{0 \mathrm{~T}}$ extracted by QSSPC from field F5 (see Fig. 1) is displayed showing excellent values ranging from 2 to $10 \mathrm{fA} / \mathrm{cm}^{2}$, indicating a wide spectrum of tools and manufacturers being capable of producing high quality layers and therefore excellent surface passivation. The extraction method used by Kimmerle et al. [6] is based on the Kane and Swanson method by fitting the lifetime in high-level injection and additionally taking into account finite carrier diffusion length and band gab narrowing.

It must be noted that such low absolute values as measured by ISE and others are significantly prone to uncertainties or inhomogeneities such as systematic errors from the measurement tool (WCT-120 from Sinton 

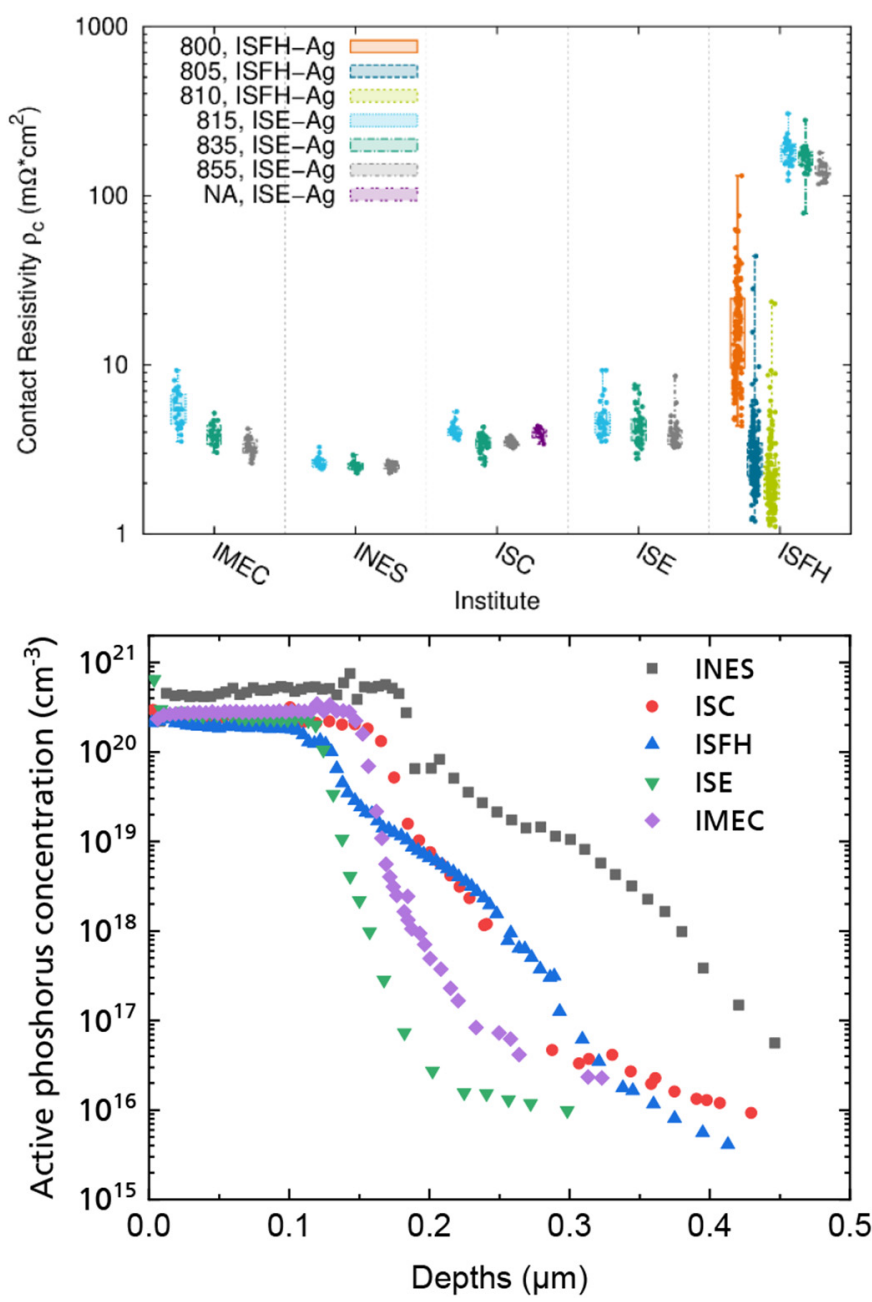

Fig. 3. The top graph shows the specific contact resistance $\rho_{\mathrm{c}}$ measured with a TLM-Scan+ device from PV-Tools for varying peak set temperature in "C". The bottom graph shows the corresponding ECV profiles for each TOPCon layer including both the phosphorus in the poly-Si (constant part) and the c-Si tail (strongly decreasing part).

Instruments). Therefore, on the upper right side the corresponding $J_{0}$ from the unmetallized reference fields F1 and F5 (infinite pitch) are shown, indicating more realistic values between 20 and $50 \mathrm{fA} / \mathrm{cm}^{2}$. Also, the $J_{0}$ from the metallized fields F3 and F8 for up to $835^{\circ} \mathrm{C}$ show comparable values to the unmetallized fields, pointing towards a true passivating contact for industrial relevant deposition and metallization technologies.

Eventually, at highest set peak temperature of $855^{\circ} \mathrm{C}$, the contact starts to depassivate, due to significant etching by the Ag paste, which is confirmed by SEM analysis. The peak set temperature range was determined with a thermal element in a way that the specimens see the same temperature profile as the final solar cells would experience.

Due to the vanishing contact recombination and frequently occurring inhomogeneities, no meaningful local $J_{0 \mathrm{~T}, \text { met }}$ can be determined.
Finally, Figure 3 completes the picture indicating a conducting contact for most of the samples with specific contact $\rho_{\mathrm{c}}$ below $10 \mathrm{~m} \Omega \mathrm{cm}^{2}$. The ISFH samples feature an additional $\mathrm{AlO}_{x}$ layer tremendously increasing $\rho_{\mathrm{c}}$, if metallized with the ISE paste ("ISE-Ag"). Obviously, that paste is not optimized for an $\mathrm{AlO}_{x}$ layer. Considering this by using a different $\mathrm{Ag}$ paste (ISFH-Ag), excellent mean values for the best group of $2 \mathrm{~m} \Omega \mathrm{cm}^{2}$ is reached. The ISFH values are obtained on-site from a different experiment on comparable layers [10].

ISC Konstanz completely finalized a part of the roundrobin samples internally, and the results are in good agreement to the ones obtained from ISE although different firing conditions are used.

Note: All TLM specimens exhibit a $n^{+} n n^{+}$-structure, hence current flow may extend in the n-bulk and the opposing unmetallized $\mathrm{n}^{+}$-layer. Depending on the tunnel resistance this might lead to an underestimation of the "real" contact resistivity on solar cell level. However, all layers are used in real solar cell devices showing high FF indicating a low tunnel contact.

Additionally, the electrochemical voltage (ECV) measured profiles for each TOPCon layer are shown. The profiles were recorded individually by each institute and provided to the main author. The doping levels are in good agreement to the contact resistivities and behave as expected. INES samples reach the lowest resistivities being also the one with the highest doping level, whereas ISE samples reach higher values due to the lower poly-Si doping.

\section{Conclusions}

$J_{0 \mathrm{~T}}$ ranging from 2 to $10 \mathrm{fA} / \mathrm{cm}^{2}$ for a single n-type TOPCon layer and $J_{0}$ ranging from 20 to $50 \mathrm{fA} / \mathrm{cm}^{2}$ for the total symmetrical device were reported. Also, for the contacted area similar $J_{0}$-values are shown pointing towards a true passivated contact. Simultaneously, low specific contact resistance well below $10 \mathrm{~m} \Omega \mathrm{cm}^{2}$ allow a high fill factor potential, if applied as rear side electrode.

All this is realized on a wide technology base using PECVD and LPCVD technology with tools from various leading equipment manufacturers such as Centrotherm, Tempress, and Semco being available in the participating institutes EPFL, CEA-INES, ISC, ISFH, IMEC and ISE. This, together with a general trend towards higher efficient solar cells considering TOPCon, clearly underlines its relevance for the PV community and industry.

It seems that the industrial passivating contact matured to a fully passivating and conducting contact enabling full efficiency potential. The fact that this can be realized using either PECVD or LPCVD from various manufacturer is expected to drive costs down and contribute to the increased adoption of TOPCon technology. This comparable to what happened earlier with the PERC technology with capital expenditures for new production lines coming down by a factor of 5 in less than 10 years due to increased competition between equipment vendors [11]. 
The authors would like to thank all people from all institutes not mentioned as authors who have been involved in the process of assembling and characterization, as well as the logistic. This work was funded by the European Union's Horizon2020 program for research, technological development and demonstration under grant agreement no. 857793 .

\section{Author contribution statement}

Tobias Fellmeth, main author: conducting experiment at ISE, coordination between institutes, writer of paper and person in charge. Frank Feldmann: coordination between institutes, helped with details in the experimental setup, proof reading. Bernd Steinhauser: a-Si deposition expert, proof reading. Henning Nagel: wetchemical expert, proof reading. Sebastian Mack; front end expert, proof reading. Martin Hermle: department leader, financial support. Frank Torregrosa: support in round robin, proof reading. Andrea Ingenito, Franz-Josef Haug, Audrey Morisset: experimental support round robin EPFL part, proof reading. Florian Buchholz, Aditya Chaudhary; experimental support round robin ISC part, proof reading. Thibaut Desrues: experimental support round robin INES part, proof reading- Felix Haase, Byungsul Min, RobbyPeibst: experimental support round robin ISFH part, proof reading. Loic Tous: experimental support round robin ISFH part, proof reading, project leader HighLite

\section{References}

1. F. Feldmann, M. Bivour, C. Reichel et al., Tunnel oxide passivated contacts as an alternative to partial rear contacts Sol. Energy Mater. Sol. Cells 131, 46 (2014)

2. C. Hollemann, F. Haase, S. Schäfer et al., 26.1\%-efficient POLO-IBC cells: quantification of electrical and optical loss mechanisms, Prog. Photovolt. Res. Appl. 26, 3 (2019)
3. D. Chen, Y. Chen, Z. Wang et al., $24.58 \%$ total area efficiency of screen-printed, large area industrial silicon solar cells with the tunnel oxide passivated contacts (iTOPCon) design, Sol. Energy Mater Sol. Cells 206, 110258 (2020)

4. A. Chaudhary, J. Hoß, J. Lossen et al., Screen printed Ag contacts for n-type polysilicon passivated contacts, AIP Conf. Proc. 2147, 40002 (2019)

5. R.A. Sinton, A. Cuevas, Contactless determination of current-voltage characteristics and minority-carrier lifetimes in semiconductors from quasi-steady-state photoconductance data, Appl. Phys. Lett. 69, 2510 (1996)

6. A. Kimmerle, J. Greulich, A. Wolf, Carrier-diffusion corrected J0-analysis of charge carrier lifetime measurements for increased consistency, Sol. Energy Mater. Sol. Cells 142, 116 (2015)

7. J.A. Giesecke, M.C. Schubert, F. Schindler et al., Harmonically modulated luminescence: bridging gaps in carrier lifetime metrology across the PV processing chain, IEEE J. Photovoltaics 5, 313 (2015)

8. D.L. Meier, D.K. Schroder, Contact resistance: its measurement and relative importance to power loss in a solar cell, IEEE Trans. Electr. Devices 31, 647 (1984)

9. F. Feldmann, M. Nicolai, R. Müller et al., Optical and electrical characterization of poly- $\mathrm{Si} / \mathrm{SiO}_{\mathrm{x}}$ contacts and their implications on solar cell design, Energy Procedia 124 $31(2017)$

10. B. Min, N. Wehmeier, T. Brendemuehl et al., $716 \mathrm{mV}$ opencircuit voltage with fully screen-printed p-type back junction solar cells featuring an aluminum front grid and a passivating polysilicon on oxide contact at the rear side, Sol. RRL 5, 2000703 (2021)

11. Y. Chen, P.P. Altermatt, D. Chen et al., From laboratory to production: learning models of efficiency and manufacturing cost of industrial crystalline silicon and thin-film photovoltaic technologies, IEEE J. Photovolt. 8, 1531 (2018)

Cite this article as: Tobias Fellmeth, Frank Feldmann, Bernd Steinhauser, Henning Nagel, Sebastian Mack, Martin Hermle, Frank Torregrosa, Andrea Ingenito, Franz-Josef Haug, Audrey Morisset, Florian Buchholz, Aditya Chaudhary, Thibaut Desrues, Felix Haase, Byungsul Min, Robby Peibst, Loic Tous, A round Robin-HighLiting on the passivating contact technology, EPJ Photovoltaics 12, $12(2021)$ 